\title{
Energieoptimierung in der Chemieindustrie
}

\section{Politisch gewollt oder wirtschaftlich notwendig?}

Die Forderung nach effizientem Einsatz von Primärenergie erfährt seit Jahren erhöhte Aufmerksamkeit. Die Diskussion um Klimaschutz, Importabhängigkeit bei Energieträgern und steigende Energiepreise, zum Beispiel auch durch den Atomausstieg, haben das Thema noch wichtiger werden lassen. Das stellt die chemische Industrie vor die Aufgabe, die Anstrengungen fortzusetzen, den spezifischen Energieeinsatz weiter zu reduzieren, auch nach Erreichen der freiwilligen Energieeinsparziele im Jahre 2012. Die Automatisierungstechnik ist hierfür ein wichtiger Hebel, um zunehmend stark integrierte Anlagen und Apparate in der Nähe des energetischen Optimums zu betreiben und die Entwicklung vom Einzel- zum Gesamtoptimum voranzutreiben.

\section{SCHLAGWÖRTER Energieeffizienz / Energiepolitik / Namur / Energiemanagement / Energieoptimierung}

\section{Energy optimisation in the chemical industry - Political goal or economic necessity?}

In recent years, public debate on the efficient use of primary energy has become more intense in view of climate change, dependency on fuel imports, and rising energy prices, linked in part in Germany to the decision to phase out nuclear power. For the chemical industry, the result is an increased focus on the reduction of power consumption beyond the self-imposed target which will be achieved in 2012. Automation offers the leverage required to operate highly integrated plants and equipment closer to the energetic optimum and to promote the development from local optimisation towards a global optimum. 
KATHARINA SCHÄCHTELE, BASF STEFAN KRÄMER, Ineos

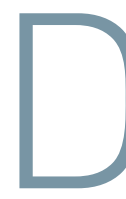

as Thema Energieeffizienz gewann in den letzten Jahren zunehmend an Bedeutung. Zuletzt rückte es durch die neuen Gesetze zur Energiewende auch politisch wieder in den Fokus. Die Namur hat Energieeffizienz bereits im Jahr 2009 als Querschnittsthema identifiziert und den Arbeitskreis 4.17 gegründet, der sich mit dem Beitrag der Automatisierungstechnik zur Energieeffizienz beschäftigt. Dieser Arbeitskreis hat die Erfahrungen aus den Mitgliedsfirmen gesammelt und beschreibt mit dem Namur-Arbeitsblatt 140 eine systematische Vorgehensweise zur Durchführung von Energieeffizienzprojekten mithilfe der Automatisierungstechnik. Da erhöhte Energieeffizienz in den Anlagen unter anderem durch eine verbesserte Prozessführung zu erreichen ist, wurden zur Namur-Hauptsitzung 2011 sowohl der politischgesellschaftliche Rahmen, als auch praktische Ergebnisse aus dem Arbeitskreis 4.17 in Kooperation mit dem Arbeitskreis 2.2 (Prozessführung) vorgestellt. Dieser Beitrag ist eine Zusammenfassung des Vortrages.

\section{WIRTSCHAFT, POLITIK UND MORAL}

Energieeffizienz ist heute ein stark politisches Thema mit einer sehr dynamischen Entwicklung, die in Deutschland durch das hohe öffentliche Interesse an Energie- und Umweltpolitik verstärkt wird. Der Ruf nach mehr Energieeffizienz leitet sich aus der Erkenntnis ab, dass die Verbrennung von fossilen Energieträgern den Klimawandel wahrscheinlich verstärkt und sich global gesehen negativ auf die Lebensbedingungen auswirkt.

Durch die stetig steigenden Preise ist Energieeffizienz aber auch wirtschaftlich ein tragender Wettbewerbsfaktor für Unternehmen, die wie die chemische Industrie global agieren und prozessbedingt einen hohen Energieverbrauch aufweisen.

Besonders in der öffentlichen Diskussion werden Begriffe und Zahlen vermischt, aber auch wirtschaftliches und moralisches Handeln zusammen genannt oder gegenseitig ausgeschlossen. Wir nähern uns dem Thema zunächst mit folgenden Fragen:
- Was heißt eigentlich Energieeffizienz und wie wird Energie auf eine neutrale Art und Weise bewertet?

- Welchen Einfluss auf dieses Thema hat die Chemieindustrie global gesehen?

- Geht es der Industrie, die Klimaziele und Einsparungen verkündet, um Ethik und Moral oder um Geld?

- Führt die augenblickliche Bewertung von Energie und Umweltbelastung dazu, dass die wirtschaftlichste Lösung auch die ökologisch-moralisch richtige Lösung ist?

Diese Fragen, die nur eine Auswahl vieler Fragen in diesem Zusammenhang darstellen, sollen als Denkanstöße dienen, das Thema aus einer übergeordneten Perspektive zu betrachten. Für den Einstieg haben wir drei plakative Beispiele aus dem täglichen Leben gewählt, die zeigen, wie komplex das Thema Energieeffizienz bei genauerem Hinsehen wird und welche Schlussfolgerungen möglich sind.

\section{Beispiel 1:}

Es in den letzten zehn Jahren gelungen, den Energieverbrauch von Kühlschränken durch bauliche Maßnahmen, wie bessere Isolierung, und durch bessere Steuerung um $60 \%$ zu senken. Dies wird durch das veränderte Energielabel (bis $2003 \mathrm{~A}$, danach A+ und A++ und seit 2011 $\mathrm{A}+++$ ) dargestellt [1]. A+++ stellt eine ein Einsparung von $60 \%$ bezogen auf A dar [2]. Zu Beginn der Effizienzskalierung dachte man, ein A-Gerät wäre das Maximum, heute liegen wir deutlich darunter. Wie weit können wir also noch kommen?

\section{Beispiel 2:}

Eine beliebte, immer wieder gestellte Frage ist, ob man beim nächsten Autokauf ein Diesel- oder Benzin-Fahrzeug erwerben sollte. Rechnet man dies beispielhaft mit den Kennzahlen aus dem Prospekt eines typischen Kleinwagens sowie KFZ-Steuer und Versicherungsprämien durch, ergibt sich, dass man nach wirtschaftlichen Kennzahlen bis zu einer Jahreskilometerleistung von $25000 \mathrm{~km}$ einen Benziner, ab $25000 \mathrm{~km}$ einen Diesel kaufen sollte. Bewertet man nach $\mathrm{CO}_{2}$-Ausstoß, sollte man immer einen Diesel fahren, da dieser einen geringeren $\mathrm{CO}_{2}$-Ausstoß hat. 
Beispiel 3:

Ein Verbraucher überlegt, welcher Apfel umweltfreundlicher ist, der aus Deutschland oder der aus Neuseeland. Die ökologische Antwort lautet, dass während der Apfelernte in Deutschland der heimische Apfel umweltfreundlicher ist, ab 6 bis 7 Monaten nach der Ernte der Apfel aus Übersee, da der Transport dann weniger Energie verbraucht, als die Lagerung des heimischen Apfels im Kühlhaus [3].

Selbstverständlich sind diese Beispiele stark vereinfacht. Aber aus Sicht des Verbrauchers, der rational nach dem individuellen wirtschaftlichen Optimum sucht, ist es nicht so wichtig, dass aus Rohöl nicht nur Diesel gewonnen werden kann oder dass man im Frühling einfach keine Äpfel essen sollte. Interessant ist jedoch, dass man aus den Beispielen Schlussfolgerungen ableiten kann, die im Kleinen ebenso wie im Großen gelten:

- Die politischen Rahmenbedingungen verzerren das Ergebnis, sodass wirtschaftliche und energetische Optimierung nicht deckungsgleich sind.

- Die Situation ist nicht jeden Tag gleich sondern kann sich mit der Zeit oder über ein Jahr so deutlich verschieben, dass verschiedene Lösungen energieoptimal sind.

Dennoch hat sich die chemische Industrie in Deutschland neben der Wirtschaftlichkeit ambitionierte Klimaund Energieziele gesetzt. So haben sich die Unternehmen vorgenommen, von 1990 bis 2012 den Treibhausgasausstoß absolut um 45 Prozent zu reduzieren und dabei den spezifischen Energieverbrauch um 35 Prozent zu senken [4]. Der gewählte Zeitraum entspricht dem Referenzzeitraum des Kyoto-Protokolls (internationales
Abkommen zur Reduktion der Treibhausgase), das im Jahr 2005 in Kraft getreten war.

Die Industrie kann sich jedoch das Setzen solcher Ziele nur erlauben, wenn sie damit den Unternehmenserfolg nicht negativ einschränkt. Denn nur wirtschaftlich erfolgreiche Unternehmen bleiben auf dem Markt bestehen und haben überhaupt erst die Möglichkeit, nach ethischen Grundsätzen zu entscheiden. Die erste wichtige Erkenntnis lautet folglich: Wirtschaftliches und ethisches Handeln schließen sich nicht aus.

Durch die unterschiedliche Bewertung von Energie und die von außen gesetzten Rahmenbedingungen kommt es allerdings vor, dass das wirtschaftliche und das energetische Optimum auseinanderfallen. Es muss deshalb das Ziel der Politik und der Unternehmen sein, ihr Handeln danach auszurichten, dass die Abweichung zwischen dem wirtschaftlichen und dem ökologisch ethischen Optimum so gering wie möglich ist.

\section{BESTANDSAUFNAHME ENERGIEEFFIZIENZ}

Um das Thema zu vertiefen, ist es nötig, auf die erste Frage einzugehen: Was ist überhaupt Energieeffizienz?

Eine Möglichkeit, Energieeffizienz von Staaten zu beschreiben, ist der spezifische Energieverbrauch pro Einheit Bruttoinlandsprodukt. Meist wird als Einheit kg Öläquivalente pro \$ Bruttoinlandsprodukt gewählt. Die Karte in Bild 1 zeigt dies für die Welt [5]. Es gilt, je heller die Blautöne, desto höher die Energieeffizienz. Europa befindet sich hier auf einem vergleichsweise hohen Effizienzniveau.

Betrachten wir jedoch den Absolutverbrauch, ergibt sich ein anderes Ergebnis. In der Karte in Bild 2 ist der Energieverbrauch in Tonnen Öläquivalent weltweit pro Bürger und Jahr dargestellt [6].
Was ist Energieeffizienz?

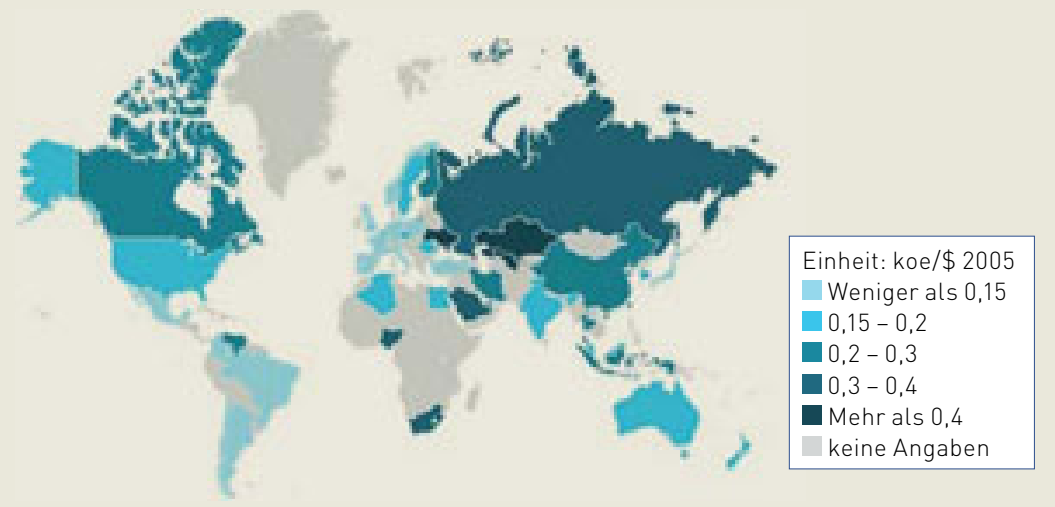

BILD 1: Energieeffizienz in kg Öläquivalent pro Dollar BIP (2010). Quelle: Enerdata

\section{Wie viel Energie brauchen wir?}

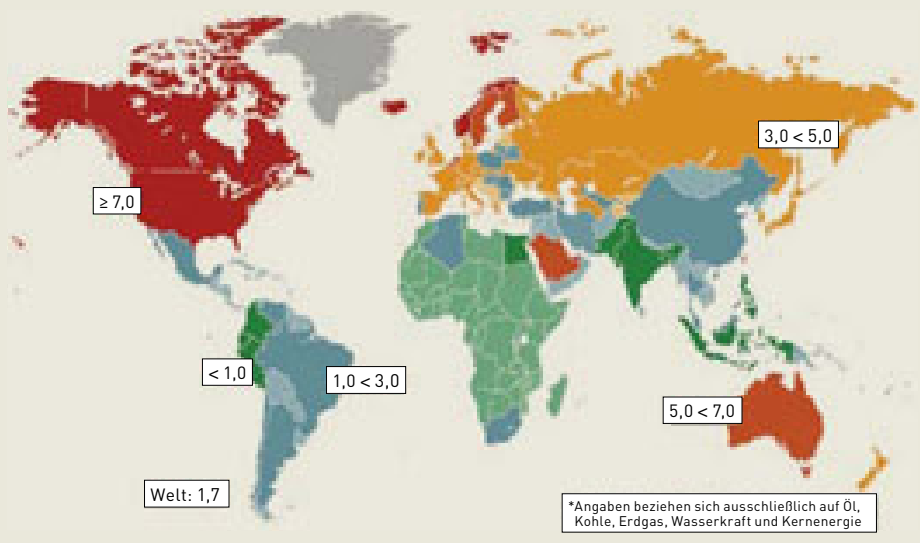

BILD 2: Energienutzung in Tonnen Öläquivalent pro Kopf pro Jahr. Quelle: Bundeszentrale für politische Bildung/Referenzjahr 2007 
Verschiedene Aspekte kommen damit zum Ausdruck:

- Die entwickelten Industrieländer im Norden haben einen deutlich höheren Energieverbrauch als der weniger entwickelte Süden. Nordamerika hat pro Kopf den höchsten Energieverbrauch. Für unseren Lebensstandard brauchen wir also pro Kopf viel Energie.

- Andere Teile der Welt wollen und müssen aufholen und werden absolut gesehen deutlich mehr verbrauchen, selbst wenn der Pro-Kopf-Verbrauch geringer bleiben sollte. Dies wird zu einem starken absoluten Anstieg des Primärenergieverbrauchs führen.

- Der augenblickliche Schnitt für die Welt liegt bei 1,7 Tonnen pro Person. Auch wenn es weltweit nur 4 Tonnen werden (Niveau Europa), werden wir den Energiebedarf mit heutigen Mitteln nicht nachhaltig decken können.

Die Schlussfolgerung ist, dass wir effizienter werden müssen, nicht nur in Deutschland sondern überall. Die deutsche Chemieindustrie schafft es (Zahlen bis 2009) eine Vorreiterrolle einzunehmen. Die Ziele, die sie sich gesetzt hat, also die in Abschnitt 1 dargestellte Reduktion des Treibhausgasausstoßes um $45 \%$ und des spezifischen Energieverbrauchs um $35 \%$, sind erreicht worden. Die vom Verband der Chemischen Industrie erhobenen Daten zeigt Bild 3 [7].

Investitionen in moderne Kraftwerke und energieeffizientere Prozesse haben die Effizienz in der Chemieindustrie deutlich verbessert. An großen Standorten ist der Verbund, also die stoffliche und energetische Integration von Prozessen, ein bedeutendes Element. Die Automatisierungstechnik trägt hier besonders dazu bei, die stark verkoppelten Prozesse auch bei zunehmender Komplexität betreibbar zu machen. Bei der BASF wurde berechnet, dass der Energieverbund bis zu 1,5 Mio Tonnen
Rohöläquivalent pro Jahr einspart [8], unter anderem weil $50 \%$ der Dampferzeugung aus Abhitzenutzung und Verbrennung von Abfällen stammt. Anzumerken ist auch, dass die heftige Umstrukturierung der Industrie in den ostdeutschen Bundesländern besonders in den 90er Jahren positiv zu diesen Zahlen beigetragen hat. In Zukunft wird auch der Ersatz von fossilen durch nachwachsende Rohstoffe zur Effizienzsteigerung beitragen, wo dies sinnvoll ist.

Ähnlich wie bei der Energieeffizienz, konnten auch die Treibhausgase aus der Produktion von 1990 bis 2009 um $45 \%$ gesenkt werden, während der Produktionsindex immer weiter zugenommen hat (Ausnahme ist die Krise im Jahr 2008) [9]. Insgesamt ergibt sich für Deutschland unter Berücksichtigung energiebedingter direkter und indirekter $\mathrm{CO}_{2}$-Emissionen plus $\mathrm{N}_{2} \mathrm{O}$-Prozessemissionen ein Anteil von $5 \%$ der Treibhausgase durch die Chemieindustrie [10].

Neben den Zahlen über produktionsbedingte Treibhausgasemissionen der Chemieindustrie sind die Ergebnisse einer Studie des International Council of Chemical Associations zu betrachten [11]. Nach dieser Studie vermeidet die Chemieindustrie über den gesamten Lebenszyklus von Produkten weit mehr Treibhausgase, als bei der Produktion anfallen. Das Verhältnis für die in der Studie betrachteten Produkte lag bei etwa 1:2,3. Dies wird in Bild 4 anschaulich zusammengefasst.

Insgesamt lässt sich also feststellen, dass die Chemieindustrie bereits heute auf einem guten Weg ist, ihren „Carbon Footprint" zu verringern und durch ihre Produkte den Klimaschutz unterstützt. Gleichzeitig ist eine weitere Effizienzsteigerung ein Wettbewerbsvorteil und eine Leistung für die Gesellschaft. Die Chemieindustrie wird deshalb ihre Energieeffizienz weiter steigern und den Ausstoß von Treibhausgasen verringern. Allgemein lassen sich

\section{Die deutsche Chemieindustrie erreicht Ziele}

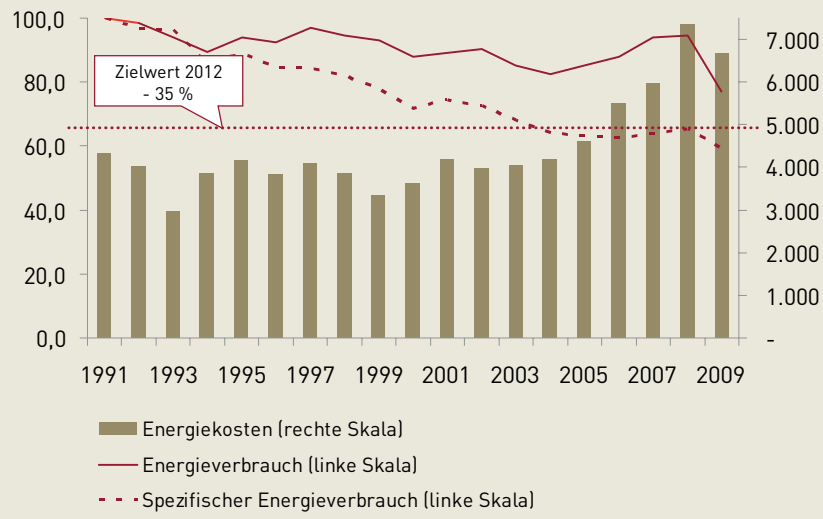

BILD 3: Energieverbrauch der deutschen Chemieindustrie von 1991 bis 2009 (1991=100), Energiekosten in Mio. Euro. Quelle: VCI
Die Chemieindustrie fördert Klimaschutz

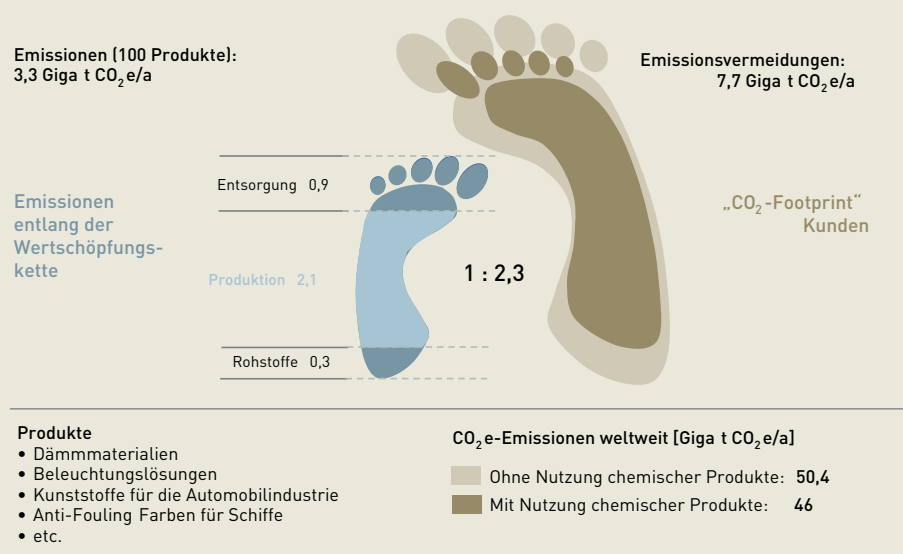

BILD 4: Klimaschutzförderung durch die Chemieindustrie. Quelle: Studie ICCA 2009 
Energieoptimierungsprojekte ähnlich angehen wie jedes andere Verbesserungsprojekt.

\section{ENERGIE- UND PROZESSOPTIMIERUNG}

Wir betrachten den allgemeinen Ansatz eines Optimierungsprojekts für einen verfahrenstechnischen Prozess in Form von 4 Stufen. Bild 5 stellt die 4 Stufen dar:

- Stufe 1 ist die Verbesserung von bestehenden Prozessen mit dem vorhandenen Equipment.

- Stufe 2 enthält zusätzlich Anlagenänderungen, was Umbaumaßnahmen und den Austausch von einzelnen Komponenten beinhaltet. Der Betrieb ist hier bereit, in verfahrenstechnische Lösungen zu investieren.

- In Stufe 3 wird eine Anlage komplett neu auf dem Papier geplant. Energieeffizienz kann jetzt am Reißbrett mit eingeplant werden, was nicht heißt, dass man nach zehn Jahren Betrieb nicht wieder bei Stufe 1 ankommt.

- Stufe 4 hieße, alles zu ersetzen, entweder das Produkt oder das komplette Verfahren der Herstellung eines Produktes.

Durch diese Stufen hindurch bewegen sich die Techniker und Ingenieure dem möglichen Optimum entgegen, wobei die Komplexität der Aufgabe und die benötigte Investition von Stufe 1 nach Stufe 4 zunehmen. Dafür nimmt die Anzahl der Projekte für die Automatisierungstechnik von Stufe 4 zu Stufe 1 zu. Die Verbesserung von bestehenden Prozessen ist daher für die Automatisierungstechnik der typische Projektfall und in der Praxis der Haupthebel zur Verbesserung der Prozessführung.

\subsection{Stufe 1: Verbesserung der Fahrweise}

Bayer MaterialScience hat als Methodik zur Energieoptimierung eine Verlustkaskade entwickelt, mit der sich die Stufen erklären lassen (siehe Bild 6).

Die Verlustkaskade zeigt auf, an welchen Stellen im Prozess Energieeffizienz verloren geht und wo das energetische Optimum liegt. Die zwischen Optimum und Ist-Zustand entstehende Differenz drückt die Verluste aus, die dynamische Verluste oder statische Verluste sein können. Dynamische Verluste entstehen zum Beispiel durch eine suboptimale Fahrweise eines Prozesses oder Fouling in Apparaten.

In der ersten Stufe der Energieoptimierung versucht man genau diese Verluste durch eine optimierte Prozessführung zu vermeiden. Sie zielt darauf ab, die bestehende Anlage optimal zu betreiben, zum Beispiel durch eine verbesserte Koordination von Energiequellen bis hin zu einem anlagenweiten Regelungskonzept, das aus OnlineDaten den optimalen Betriebspunkt bestimmt und in den Betrieb der Anlage eingreift.

In petrochemischen Prozessen lässt sich dies für große Kontianlagen durch modellbasierte prädiktive Regelung (MPC) erreichen, wie das Beispiel zeigt. Die MPC Regelung ermöglicht durch eine Optimierungsrechnung unter Berücksichtigung der Prozessdynamik, dass der Prozess an seiner optimalen Grenze gefahren wer- den kann und, sollten Störungen oder eine Verschiebung des Optimums auftreten, auch gut wieder dorthin bewegt werden kann.

In diesem Beispiel muss durch das Anfahren der Qualitätsgrenze im Absorberkopfstrom und der Optimierung der Reaktionsbedingungen im Reaktor der Stripper nicht mehr so viel Arbeit leisten und kann damit Dampf sparen.

\subsection{Stufe 2: Verbesserung durch Anlagenmodifikation}

Während sich Stufe 1 komplett über Maßnahmen aus der Prozessführung realisieren lässt, ist Stufe 2 mit Umbauten verbunden. In der in Bild 6 gezeigten Verlustkaskade entspricht diese Optimierungsstufe der Reduktion von statischen Verlusten. Um weiter in Richtung des theoretischen Optimums zu gelangen, müssen diese statischen Verluste, die durch ein suboptimales Apparatedesign entstehen, reduziert werden. Dafür wird überholtes Equipment ausgetauscht oder Anlagenteile, für die es modernen Ersatz gibt, werden umgebaut. Dabei stehen häufig Ziele wie Kapazitätserweiterung, verbesserte Wärmeintegration oder effizientere Apparate im Vordergrund.

Die geplanten Änderungen geben in vielen Fällen der Prozessführung ein Fenster, weitere Verbesserungen vorzunehmen. Oft sind die Neuerungen auch nur durch eine Verbesserung der Prozessführung betreibbar.

Dabei ist sehr wichtig, dass die Anlagenplaner und die Prozessführer sehr frühzeitig zusammenarbeiten. Wir sind der Meinung, dass diese Art von Aufgabenstellung durch die interdisziplinäre Arbeitweise der Energieoptimierungsteams zunehmen wird.

Ein klassisches Beispiel aus der Energieoptimierung, mit dem durch einen vergleichsweise kleinen Umbau eine große Einsparung erzielt wird, ist die zusätzliche Nutzung von Frequenzumrichtern bei Pumpenmotoren. Anstatt von Stellventilen wird die benötigte Durchflussmenge durch die Variation der Pumpendrehzahl reguliert. Es gilt die Faustregel, dass eine Halbierung der Fördermenge mit Drehzahlregelung einer Reduktion der Leistung auf etwa ein Achtel entspricht. Ein weiterer Schritt wäre auch der Ersatz von älteren, weniger energiearmen Motoren durch neuere, wesentlich energieeffizientere Motoren.

\subsection{Stufen 3 und 4: Verbesserung durch Anlagenneubau oder Produktaustausch}

Die größten Potenziale, aber auch die höchsten Investitionen ergeben sich, wenn Anlagen neu geplant werden oder sogar völlig neue Anlagentypen entwickelt werden, weil ein neues Verfahren oder neue Produkte erforderlich sind. Für die Energieeffizienz kommen auf Apparateseite dann modernste Technologien zum Einsatz.

Die Ingenieure aus der Prozessführung müssen solche Aufgabenstellungen sehr selten lösen. Die Herausforderung ist wie in Stufe 2 eine frühzeitige Zusammenarbeit mit der Planung, damit die richtigen Technologien mit den entsprechenden Schnittstellen ausgewählt werden. Dann ergeben sich zusätzliche Möglichkeiten, wie zum Beispiel über 


\section{Stufenweise Optimierung}

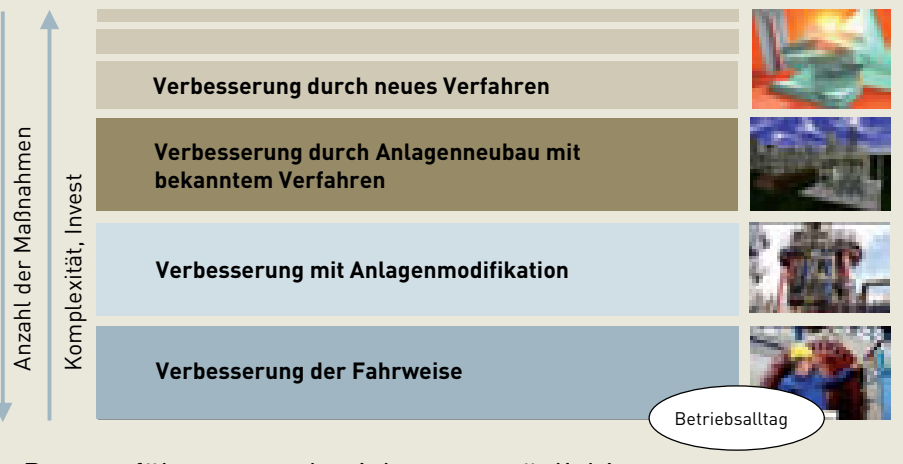

Prozessführung macht vieles erst möglich!

BILD 5: Stufenweise Optimierung einer

Produktion, allgemein dargestellt

\section{Stufe 1: Verbesserung der Fahrweise}

- Betriebspunktoptimierung

- Koordination von Energieerzeugern und -verbrauchern

- Anlagenweite Regelung (MPC, RTO)

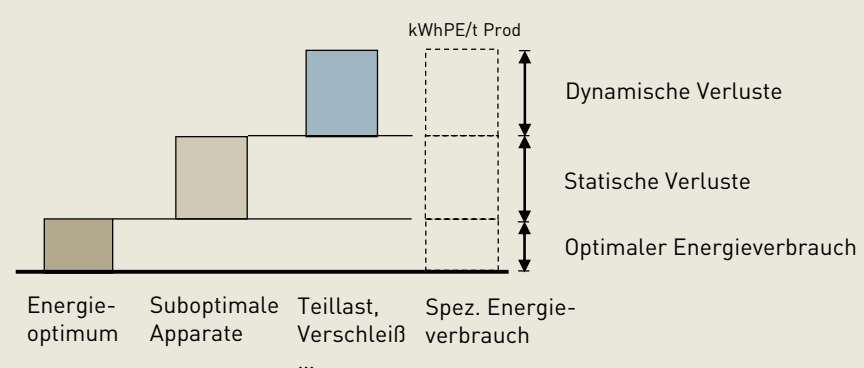

BILD 6: Verlustkaskade einer chemischen

Produktion Quelle: Bayer MaterialScience

\section{Stufe 1: Verbesserung der Fahrweise}

Beispiel: Modellprädiktive Regelung

- Ein Modell „kennt“ die Prozessdynamik und Prozessgrenzen

- Die modellprädiktive Regelung plant damit optimale Sollwerte für unterlagerte Regler

- Anwendung: Reaktor mit Absorber-Stripper Prozess

- MPC optimiert Reaktionsbedingungen und minimiert den Dampfeinsatz im Stripper unter Ausnutzung der Qualitätsgrenzen im Recyclestrom Energieoptimal!
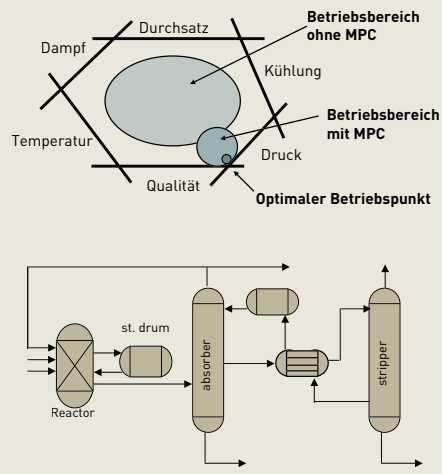

BILD 7: Prinzip der Modellgestützen Prädiktiven Regelung mit vorgeschalteter linearer Optimierung Quelle: BASF

\section{Wo liegt eigentlich das Optimum?}

\author{
Markt \\ - Preise \\ - Verträge \\ - Politische Einflüsse
}

- Verbund

- Austausch zwischen Anlagen

- Standortweite Dampfnetze

- Intelligente Kraft-Wärmekopplung

- Anlage

- Wärme-Integration

- Prozessintensivierung

- Apparat

- Apparatedesign

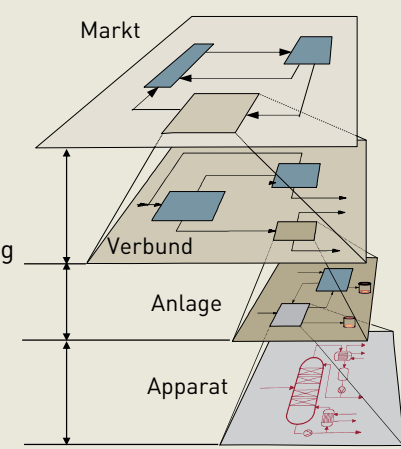

BILD 8: Vom Apparat zum Verbund:

Die verschiedenen Ebenen der Komplexität
Trainingssimulatoren, das Prozessführungskonzept offline zu erproben und Anlagenfahrer frühzeitig zu schulen.

Beispiele für die Stufe 3 und 4 sind das Verfahren zur direkten Oxidation für Propylenoxidherstellung (-35 \% Energieverbrauch [12]), die Ablösung der Chlorherstellung mittels Quecksilberelektrode oder das Verbot von FCKW und die Entwicklung von Ersatzstoffen.

\subsection{Komplexität der Energieoptimierung}

Das Stufenmodell wirkt sehr einfach, dennoch wird der Kunde immer wieder feststellen, dass die Details zu hoher Komplexität führen. Diese Komplexität entsteht aus den vielen Verkopplungen in und zwischen einzelnen Chemiebetrieben.

Wenn versucht wird, ein Optimum zu bestimmen, muss der Geltungsbereich definiert werden. Es gibt ein Optimum für einen Apparat, eine Anlage oder einen Standort. Das Standort-Optimum, welches durch die vielen Verknüpfungen ungleich schwerer zu finden ist, mag dazu führen, dass einzelne Betriebe oder Apparate nicht am Optimum gefahren werden, was dann dem Anlagenpersonal vor Ort kaum zu erklären ist. Das heißt auch, die Summe der Einzeloptima entspricht nicht zwingend dem Gesamtoptimum. Außerdem verändert sich dieses Opti- 
Praktische Ansätze zur Energieoptimierung

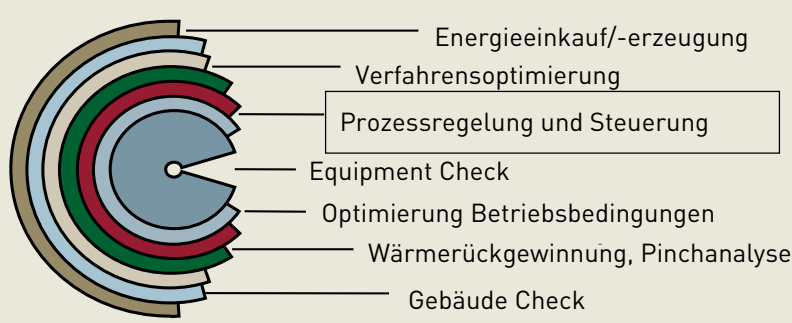

- Welche Methoden und Maßnahmen?

- Welche Chancen und Hemmnisse gibt es?

- Wie wird die Wirtschaftlichkeit bewertet?

BILD 9: Zwiebelprinzip der Energieoptimierung Quelle: Bayer Technology Services

\section{Erfassung und Bewertung des Ist-Zustands}

Beispiel: Jahreslastgang Kühlsole

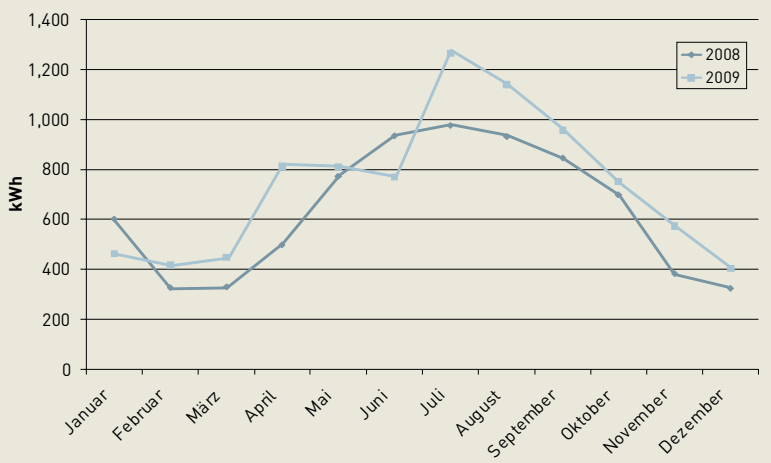

BILD 10: Veränderung des Energienutzens über ein Jahr am Beispiel des Jahreslastgangs von Kühlsole Quelle: Sanofi Aventis mum je nach Betriebsbedingungen, Rohstoffqualität, Kundenanforderungen und anderen Umgebungsbedingungen.

Es gibt darüber hinaus aber auch noch Energieverträge, gesetzliche Rahmenbedingungen und Preisschwankungen auf dem Energie- und Rohstoffmarkt, die das Optimum zusätzlich in die eine oder andere Richtung verschieben, wodurch mathematisch gesprochen ein nichtlineares, gemischt-ganzzahliges Optimierungsproblem entsteht, das höchst schwierig zu lösen ist.

Die Automatisierungstechnik bietet die Möglichkeit, die nötigen Informationen zu bündeln und Komplexität herauszunehmen und die Richtung für einen wirtschaftlich optimalen Betrieb vorzugeben.

Letztendlich muss Energieeffizienz im Alltag beim Betriebspersonal ankommen und für die Betriebsmannschaft einfach zu bedienen sein. Das heißt, wir müssen die Ebenen abbilden und wo möglich dem Betriebspersonal auf Basis einer Gesamtrechnung eine optimale Fahrweise vorschlagen, denn es bringt keinen Vorteil, wenn das Optimum nicht angefahren wird, weil die Anlagenfahrer die Regler ausschalten. Die moderne Prozessführung macht dies möglich.

\section{PRAKTISCHE ANSÄTZE ZUR ENERGIEOPTIMIERUNG}

Die Namur hat Energieeffizienz frühzeitig als Querschnittsthema identifiziert, da sie erkannt hat, dass die Automatisierungstechnik dazu einen wichtigen Beitrag leistet. Sie ist die Basis dafür, dass Prozesse trotz der Veränderungen von Betriebspunkten und Störungen im Prozess sicher und stabil laufen, während Prozessgrenzen ausgenutzt werden. Ihr Ziel ist es, den Betrieb für den Bediener zu vereinfachen und dabei den Abstand zwischen dem Betriebspunkt und dem Optimum über die Zeit so gering wie möglich zu halten. Darüber hinaus entstehen besonders viele Innova- tionen durch die Schaffung von Schnittstellen zur Prozessentwicklung, Anlagenplanung und zu den Betreibern der Anlage.

Auf Basis der bisher genannten Erkenntnisse wurde der Leitfaden des AK 4.17 Energieeffizienz entwickelt. Der Arbeitskreis 2.2 „Prozessführung“ unterstützte den Arbeitskreis 4.17 durch die Entsendung von Mitgliedern und stand beratend für den Bereich der optimalen Prozessführung zur Seite. Das Arbeitsblatt nennt sich Vorgehensweise zur „Steigerung der Energieeffizienz - Beitrag der Automatisierungstechnik" und wird derzeit für die Veröffentlichung vorbereitet. Das Dokument richtet sich an den Praktiker, der betriebliche Energieeffizienzmaßnahmen plant und umsetzt. Dabei kann der Leitfaden als Einstieg dienen; er wird allerdings niemals den Experten mit seinem Fach- und Erfahrungswissen ersetzen.

Fest steht, dass Prozessregelung und Steuerung nicht der einzige Hebel für Effizienzsteigerungen sind. Zu den bekanntesten Ansätzen gehören sicherlich verfahrenstechnische Maßnahmen, wie die Wärmeintegration und Wirkungsgradverbesserungen. Außerdem ist die regelmäßige Überprüfung und Wartung von Komponenten unverzichtbar, damit sich das Effizienzniveau mit der Zeit nicht verschlechtert. Auch der Faktor Gebäudeenergieverbrauch rückt aufgrund steigender Kosten immer häufiger in den Fokus der Energiemanager. Schließlich sind auf der Seite der Energieerzeugung und des Einkaufs Energieoptimierungen möglich, die sich auf die Produktion auswirken. Vor dem Hintergrund, dass die Chemieindustrie in den letzten Jahren ihre Aktivität ausgebaut hat, können verbleibende Potenziale vor allem durch interdisziplinäre Ansätze identifiziert und umgesetzt werden.

Die Vorgehensweise zur Ermittlung von Potenzialen und Maßnahmen zur Steigerung der Energieeffizienz ist allgemeingültig. Sie lässt sich als iterativer Prozess beschreiben und teilt sich in fünf Phasen: 


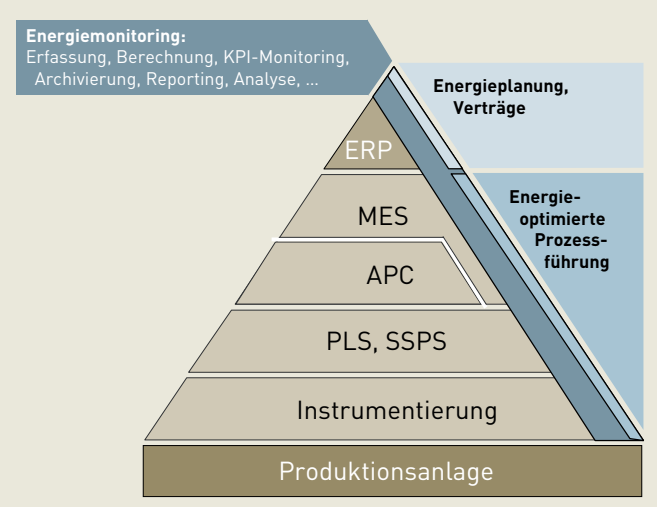

BILD 11: Um Energiemonitoring und Energieoptimierung erweiterte Automatisierungspyramide Quelle: BASF
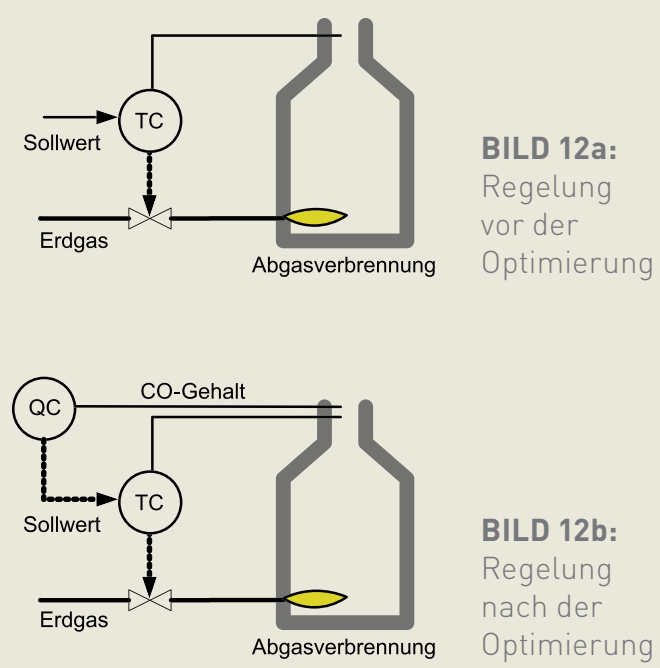

- Aufnahme des Ist-Zustands

- Grobanalyse

- Feinanalyse

- Bewertung und Maßnahmenauswahl

- Umsetzung und Erfolgskontrolle

Die Herausforderung bei der Erfassung und Bewertung des Ist-Zustands liegt darin, den Energieverbrauch für jeden Energieträger (zum Beispiel Dampf, Erdgas, Strom) und jeden Prozessschritt über die Zeit zu messen. Um eine Aussage über die Anlageneffizienz zu treffen, müssen diese Zahlen in Abhängigkeit zur jeweiligen Produktion (beispielsweise Produktionsmenge) gesetzt werden. Erst damit kann ein Effizienzvergleich über verschiedene Betriebspunkte oder mit ähnlichen Anlagen stattfinden. Die Realität sieht dagegen oft so aus, dass der Gesamtverbrauch eines Energieträgers über längere Abrechnungszyklen am Baueingang abgelesen und dem Betrieb in Rechnung gestellt wird. Es gilt also nicht nur, vorhandene Daten zu sammeln, sondern meistens müssen auch zusätzlich Datenlücken geschlossen werden. Beispielhaft zeigt der Jahreslastgang für eine Kühlsole in Bild 10, wie die Datenerhebung aussehen kann.

Die unterschiedlichen Verbräuche im Verlauf des Jahres, sowie zwischen den Jahren, werden deutlich sichtbar und müssen in der weiteren Analyse auf unnötige Effizienzverluste untersucht werden.

Nach der ersten Übersicht des Energieverbrauchs erfolgt eine Priorisierung der Energieverbraucher nach der Höhe der vermuteten Potenziale. Für die Auswahl werden dann in den Phasen Grob- und Feinanalyse die Ursachen für Effizienzverluste immer genauer bestimmt, Optimierungspotenziale berechnet und alternative Lösungsvorschläge entwickelt. An dieser Stelle ist die Zusammenarbeit von Experten aus der Vielzahl der genannten Disziplinen besonders nachhaltig, um die richtigen Schwerpunkte zu setzen und gemeinschaftliche Lösungen zu entwickeln.
Für die Steigerung der Energieeffizienz von chemischen Anlagen durch eine verbesserte Prozessautomatisierung ergeben sich zwei Schwerpunkte: das Energiemonitoring und die energieoptimierte Prozessführung (siehe Bild 11). Alle Ansätze haben zum Ziel, den Wirkungs- und Nutzungsgrad von Anlagen zu erhöhen, und damit den spezifischen Energieverbrauch zu reduzieren.

Maßnahmen aus dem Bereich des Energiemonitorings tragen dazu bei, den Zustand der Anlagenkomponenten zu überwachen und Fehler frühzeitig zu erkennen. Alterung, Verschleiß und Verschlechterung des Wirkungsgrades verursachen ein stetiges Wegdriften der Anlagenparameter und verringern unter Umständen die Standzeit der Gesamtanlage und deren Energieeffizienz erheblich. AuBerdem hilft die kontinuierliche Messung des Energieverbrauchs, sich ändernde Rahmenbedingungen zu erkennen und die Anlage näher am Energieoptimum zu betreiben.

Auch die Energieplanung und das Vertragsmanagement sind Teil des Energiemonitorings und werden mit Produktionsdaten aus den unteren Ebenen der Automatisierungspyramide unterstützt. An dieser Stelle sollten vor allem geeignete Anreize geschaffen werden, um die Energieeffizienz des Unternehmens langfristig zu steuern.

Maßnahmen, die direkt in den Produktionsprozess eingreifen, gehören zur Kategorie der energieoptimierten Prozessführung. Dabei werden Optimierungen an Reglerkonfigurationen oder die Implementierung neuer Regelfunktionen im Prozessleitsystem durchgeführt. In besonderen Fällen werden komplexe Advanced-Process-Control-Algorithmen auch separat aufgesetzt und über entsprechende Schnittstellen mit Prozessleit- und Betriebsdatensystem verbunden. Darüber hinaus können historische Produktionsdaten aus Prozessleit- und Betriebsdatensystem zur Modellidentifikation und Entwicklung neuer Regelalgorithmen dienen. Es ist zu beachten, dass die Automatisierungs- 
technik hier die Infrastruktur zur Verfügung stellt, um entsprechende Parameter zu erfassen und zu optimieren. Eine Effizienzsteigerung, kommt demnach durch eine robuste Abbildung und Beherrschung der komplexen Prozesse zu Stande.

Im nächsten Schritt folgt die Auswahl der Maßnahmen anhand des technischen, ökonomischen und ökologischen Nutzens, wobei jedes Unternehmen eigene Ansätze verfolgt. Das Arbeitsblatt geht daher auf diesen Schritt nicht weiter ein, sondern beschränkt sich auf einen Katalog nützlicher Bewertungskriterien.

Die folgenden Beispiele finden sich zum Teil auch im Arbeitsblatt wieder.

\section{Beispiel 1 (Teilanlage):}

Abgasverbrennungskessel brauchen zumeist Zusatzbrennstoff, um das Abgas auf Solltemperatur aufzuheizen und damit erhöhte CO-Konzentrationen im Abgas zu vermeiden. Die notwenige Brennstoffmenge wird dabei über eine einfache Temperaturregelung im Abgas eingestellt, sodass die gesetzlich vorgeschriebene Abgaskonzentration an CO nicht überschritten wird.

Durch den Einsatz einer kostengünstigen CO-Sonde kann eine Reglerkaskade aufgebaut werden, die die Solltempereratur näher an die CO-Grenze führt. Mit dieser Maßnahme konnten in einer Untersuchung der BASF
Einsparpotenziale von bis zu $10 \%$ der ursprünglichen Erdgasmenge identifiziert werden [18].

\section{Beispiel 2 (Anlage):}

Ein Standort der Ineos wird mit Dampf auf mehreren Druckstufen versorgt. Der Druck im Hochdruck-Netz wird häufig über eine Überströmung in die untere Dampfstufe geregelt. Um eine ausreichende Regelreserve zu halten, wird kontinuierlich mehr Hochdruck-Dampf erzeugt als nötig. Mit dem neuen Prozessführungskonzept wird nur dann HD-Dampf übergeströmt, wenn der Druck in der unteren Stufe zu stark abfällt oder um einen Überdruck im Hochdruck-Netz zu vermeiden. Schwankungen in der Netzlast werden durch das kontinuierliche Nachfahren der Hochdruck-Dampf-Produktion reguliert.

\section{Beispiel 3 (Verbund):}

Im Anlagenverbund der Ineos können sowohl das Kraftwerk als auch mehrere Hilfskessel im Steamcracker Dampf für die Hochdruckschiene erzeugen. Die Lastverteilung zwischen den Anlagen sollte so erfolgen, dass möglichst wenig zusätzlicher Brennstoff zur Dampferzeugung eingesetzt wird. Dies ist im Beispiel durch eine Online-Optimierung gelungen, die mithilfe von Modellen für die verschiedenen Dampferzeuger unter Berücksichtigung von definierten Randbedingungen das energetische Optimum bestimmt
BILD 13a:

Regelung

vor der

Optimierung

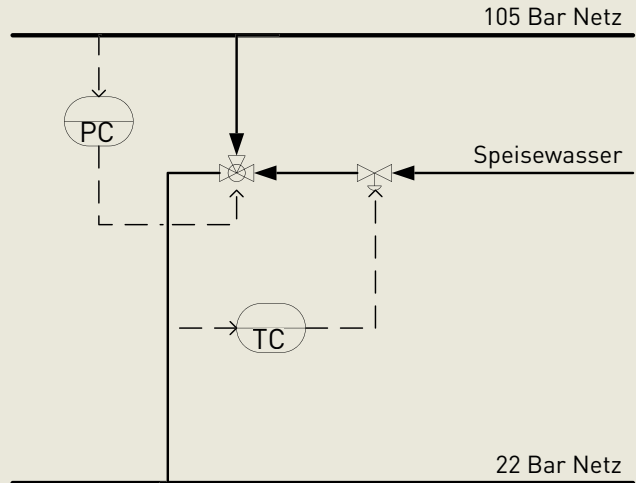

BILD 13b:

Möglichkeit

einer

Optimierung

der Regelung

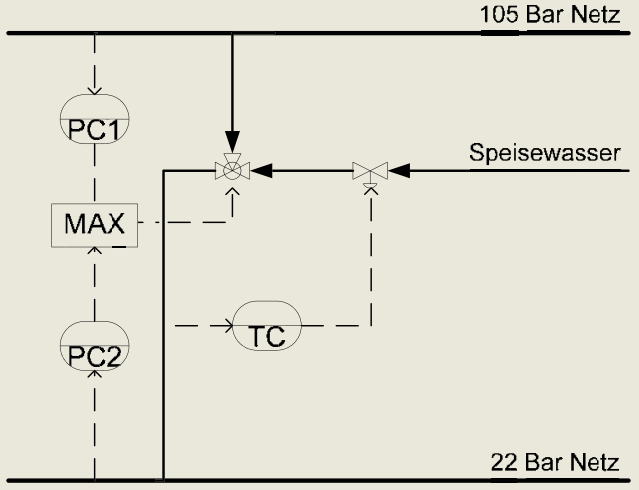

\section{REFERENZEN}

[1] Die Europäische Kommission: Richtlinie 2010/30/EU des Europäischen Parlaments und des Rates vom 19. Mai 2010.

(http://eur-lex.europa.eu/LexUriServ /LexUriServ.do? uri=0J:L:2010:153:0001:0012:DE:PDF)

[2] http://de.wikipedia.org/wiki/Energieverbrauchskennzeichnung, abgerufen am 10.2.2012.

[3] Achatz, B.: Nachhaltigkeitsbewertung mit der Ökoeffizienzanalyse (2010), interner Vortrag BASF

[4] Verband der chemischen Industrie: Weiterentwickelte Selbstverpflichtungserklärung der chemischen Industrie im Rahmen der Klimaschutzvereinbarung der deutschen Wirtschaft vom November 2000, (2001)

[5] British Petroleum (BP): Statistical Review of World Energy 2008; United Nations Population Fund (UNFPA): State of world population 2007 labgerufen über Bundeszentrale für politische Bildung: www.dpd.de/ files/JQ3RZ.PDFI

[6] Enerdata: Global Energy Statistical Yearbook 2011 Energy intensity of GDP at constant purchasing power parities, (2011). www.enerdata.net

[7] Verband der chemischen Industrie: Foliensatz Energiekosten (S.8), und persönlicher Kontakt per Email am 17.10.2011, (2011)

[8] BASF: Ressourcenschonung, (2011). http://www.basf.com/group/corporate/de/ sustainability/environment/resource-conservation/index 
und einstellt. Bemerkenswert ist hier, dass eine falsche Wahl der internen Dampfpreise zu völlig verzerrten Ergebnissen führen kann - also politische Eingriffe im Kleinen.

Nach der Umsetzung von Maßnahmen ist unbedingt eine Erfolgskontrolle notwendig, die realisierte Einsparpotenziale dokumentiert und den Betrieb beim Erreichen des Energieoptimums unterstützt. Das bereits erwähnte Monitoring ist gleichzeitig der Ausgangspunkt für Nachfolgeprojekte in der nächsten Iterationsschleife zur Verbesserung der Effizienz.

\section{SCHLUSSFOLGERUNGEN UND BOTSCHAFTEN}

Die Chemieindustrie hat sich beim Thema Energieeffizienz ambitionierte Ziele gesteckt und sie erfüllt, durch moderne Kraftwerke, Synergieeffekte im Verbund und effiziente Prozesse. Darüber hinaus tragen viele chemische Produkte dazu bei, den Treibhausgasausstoß weltweit zu reduzieren. Die Nutzung chemischer Produkte führt für ausgewählte Erzeugnisse zu einer Verringerung $\mathrm{CO}_{2}$-Emission um mehr als 50 Prozent. [10] Wirtschaft und Moral stehen hier nicht im Widerspruch, sie fördern sich gegenseitig.

Gleichzeitig konnte im Beitrag gezeigt werden, dass politische und gesetzliche Rahmenbedingungen das Gesamtoptimum beeinflussen. Die Rahmenbedingungen müssen deshalb global so gesetzt werden, dass keine unerwünschten Verzerrungen (weg vom energetischen Optimum) auftreten. Energieeffizienz muss sich wirtschaftlich lohnen, damit Unternehmen die Entwicklung weiter vorantreiben!

Die Automatisierungstechnik trägt in diesem Zusammenhang wesentlich dazu bei, die steigende Komplexität der Anlagen beherrschbar zu machen. Sie hat dazu die Aufgabe, die „Regelabweichung“ zwischen (energetischem) Optimum und Realität so gering wie möglich zu machen und bisher verdeckte Potenziale zu heben. Durch moderne Technologien ist es möglich, Informationen zwischen Systemen (wie Produktionsanlagen, Energieverbund, Markt) auszutauschen und letztendlich vom Einzeloptimum immer weiter in Richtung Gesamtoptimum zu gelangen. Die Automatisierungstechnik ist dabei ein zentraler Enabler zur Steigerung der Energieeffizienz im Umfeld der Chemieindustrie.

\section{AUTOREN}

[9] Verband der chemischen Industrie: Foliensatz Energie und Klimapolitik - Business Impact für die chemische Industrie (S.4), (2011)

[10] Verband der chemischen Industrie: Persönlicher Kontakt T. Benzig per Email am 17.10.2011, (2011)

[11] International Council of Chemical Associations: Innovations for Greenhouse Gas Reductions, (2009)

[12] Bayer Material Science: Verlustkaskade, STRUCTese ${ }^{\circledR}$ Energieeffizienz Programm (2010)

[13] BASF: Interner Vortrag Modellprädiktive Regelung, (2011)

[14] BASF: BASF und Dow erhalten US-amerikanischen Umweltschutzpreis für ihre HPPO-Technologie, (2010). http://www.basf.com/group/pressemitteilungen/ P-10-327

[15] BASF: Interner Vortrag Energieeffizienz, (2011)

[16] Bayer Technology Services ( A. Jupke, H.-J. Leimkühler): Nachhaltige Implementierung von Energie- und Klimaeffizienz (Übersichtsvortrag), ProcessNet-Jahrestagung, Mannheim, 8. - 10. September 2009, Kurzfassung in: Chemie Ingenieur Technik 81 (8), S. 1106, 2009

[17] Namur 2011: NA140, Vorgehensweise zur Steigerung der Energieeffizienz in chemischen Anlagen (Entwurf), S.19-21, 2011

[18] BASF Fachzentrum Automatisierungstechnik: Steigerung der Energieeffizienz von Produktionsanlagen durch eine Optimierung der Prozessführung, (Vortrag 0 . Kahrs beim Jahrestreffen der Fachgemeinschaft PAAT 2009]

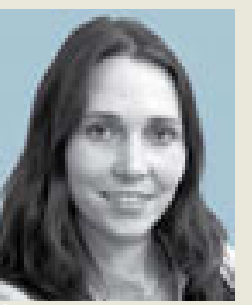

M.Sc. KATHARINA SCHÄCHTELE (geb. 1981) begann nach ihrem Master in Process Energy Environmental Systems Engineering an der TU Berlin im Jahr 2010 bei BASF. Dort wurde sie als Ingenieurin im Fachzentrum für Automatisierungstechnik angestellt. Seit 2011 ist sie im Stab Centers of Technical Expertise tätig.

BASF SE,

GTF - L440, D-67056 Ludwigshafen,

Tel. +49(0) 6216094651 ,

E-Mail: katharina.schaechteleabasf.com

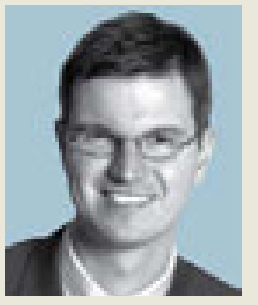

Dr.-Ing. STEFAN KRÄMER (geb. 1972), ist Ingenieur für Verfahrens- und Chemietechnik und hat an den Universitäten Newcastle (GB) und Dortmund in Anlagensteuerungstechnik studiert beziehungsweise promoviert. Seit 2009 ist er bei Ineos Köln als Teamleiter in der Gruppe Process Control und Application Engineering beschäftigt.

Ineos Köln GmbH,

Alte Straße 201, D-50769 Köln,

Tel. +49 (0) 221355526578 ,

E-Mail: stefan.kraemer@ineos.com 